\title{
ACUTE AND CHRONIC RETENTION
}

\section{By ARTHUR JACOBS, F.R.F.P.S.Glasg. (Surgeon, Urological Dept., Glasgow Royal Infirmary)}

By acute retention is meant a sudden and complete inability to pass urine. A chronic retention means that the patient is unable to empty the bladder, though micturition is still possible, and may even be apparently quite free. The quantity unevacuated from the bladder is termed residual urine, and this may vary in amount from a few ounces in cases of minor obstruction, to two or more pints in advanced manifestations. The two phases may merge from one to the other. Thus an acute retention may supervene on a previously established chronic retention and revert back to the latter category following relief of the acute attack.

Whilst the diagnosis of acute retention is self-evident, that of chronic retention may not, at first, be apparent. Diminution in the force of the stream and urinary frequency are the outstanding symptoms which suggest that the bladder is not being completely emptied. Suprapubic palpation and percussion may confirm the diagnosis providing the abdominal wall is not too obese. Confirmation is definitely obtained by passing a catheter, after the bladder has been emptied to the best of the patient's ability. If a large residual is present, not more than eight to ten ounces should be allowed to run off at such a diagnostic test.

It should be borne in mind that advice is frequently sought from patients with a chronic vesical distension, who complain of little or no disturbance directly referable to the bladder. A gradually increasing capacity, resulting from atony of the bladder musculature, may relieve the patient of any need for frequent emptying, even in the presence of a very large residual. As a result of back pressure effects on the upper urinary tract, however, and the consequent renal impairment that follows, gastro-intestinal and cardio-vascular disturbances arise, and the clinical picture may, at first sight, obscure the underlying causative lesion. The significance of these symptoms in relation to chronic vesical distension will be more fully discussed subsèquently, for they comprise a group who frequently have a grave prognosis.

Retention, like haematuria, is a symptom, and the causes are variable. The male is, of course, the more frequent victim by reason of his genital anatomy, but women and children may also suffer.

The following classification is offered as including most of the lesions responsible for retention, acute or chronic:-

Obstructive: Prostatic enlargement (benign and malignant); prostatic abscess and prostatis; fibrous obstruction at the vesical outlet; urethral stricture; blood clot; vesical and urethral calculus; rupture of bladder and urethra; foreign body in bladder and urethra.

Non-obstructive: Neurogenic or cord bladder; post-operative; hysteria.

Retention in the Female: Impacted uterine fibroids; malposition of gravid uterus; all the lesions listed in the first two sections excepting those associated with the prostate.

Infants and Children: Imperforate prepuce and meatus; meatal contracture; atresia of the urethra; congenital valve or cyst in posterior urethra; all lesions listed in first two sections excepting those associated with the prostate.

\section{ACUTE RETENTION}

It is necessary to make a diagnosis of the cause of an acute retention before proceeding to deal with it; as the methods to be employed for relieving the patient vary considerably in different instances. In the following sections the principal causative lesions are separately discussed. The obstructing prostate and stricture of the urethra account for more than 90 per cent of all cases in the male.

\section{The Obstructing Prostate.}

This is the commonest cause of acute retention. There may be a history of earlier prodromal symptoms, such as nocturnal frequency and diminution in the force of the stream, or the retention may be the first intimation to the patient that he has an enlarged prostate. Excess of alcohol, exposure to cold, or deliberately retaining the urine for a period long after the desire to micturate has been felt, are common predisposing factors for sudden retention in this latter group, particularly when the prostate is of the large soft succulent variety. The age, history of the onset, and a rectal examination will generally suffice to confirm the cause of the retention. 
The patient is usually in great distress, and immediate relief is called for. His distress is frequently aggravated by the mental anxiety of wondering what is going to happen if he fails to get rid of his urine. Thus a preliminary reassurance that he is not in danger may help to calm the insistent call for immediate relief and avoid the attending doctor from being stampeded into a hasty and ill-advised procedure. If the patient is of the robust type and is well enough to be placed in a hot bath, sitting there for fifteen minutes may relieve the congestion and oedema in the prostate and allow a gradual voiding of urine to take place. The bladder may empty itself, some of the urine only, may come away, or no relief at all may follow. In that event catheterisation is indicated. The instrument of first choice should be the soft rubber catheter, as it produces least trauma. If this fails to negotiate the obstruction, a semi-rigid gum elastic Coudé catheter, with an angle of 30 to 35 degrees, and a gauge of I4 to I 8 Charriere, should next be tried. With care and skill few failures should follow with this type of catheter. Only as a last resort should the overcurved metal catheter be employed. Although the passing of a urethral catheter is in itself a minor surgical procedure, it is not unattended by danger, particularly in the prostate patient, and especially so in the presence of established renal or cardiac disease. Infection, trauma, and shock or haemorrhage, from too hasty relief may adversely influence the patient's whole future outlook, and even cost him his life. Such hazards can be reduced to a minimum by scrupulous asepsis, avoidance of trauma, and gradually emptying a bladder that is grossly distended. The subject of gradual decompression will be further discussed under chronic retention. The following points on the technique of passing a urethral catheter may prove useful:-

The hands and instruments should be surgically clean. The glans should be cleansed with a mild antiseptic such as boric acid ( 2 per cent) or potassium permanganate ( $I$ in 8,000). The same solution can be employed to gently irrigate the urethra, using a urethral syringe for this purpose. A catheter of a size that will easily pass through the external meatus should be chosen and gently pushed forwards along the urethra until the bladder is reached. In the absence of any organic obstruction, the usual points where a hold-up may be encountered are at the junction of the bulbous and membranous urethra and at the vesical outlet. As ideal aseptic precautions for catheterisation may be difficult tos achieve outside of an operating theatre, the writer favours the use of gum elastic catheters, previously sterilised and kept ready in a formalin vapour container, in circumstances such as might be encountered in having to deal with an acute retention in the patient's own? home. Catheters of this type are easily introduced without manually contaminating any portion going into the bladder. It is, of course, possible to feed into the urethra a soft catheter, which is grasped by sterile forceps, and thereby avoid touching any portion other than the outer end.

Patients suddenly seized with acute retention due to prostatic obstruction are quite frequently able to resume normal micturition after one or more catheterisations. The question of dealing with the offending prostrate either by prostatectomy or perurethral resection should, however, be considered, for recurring attacks can generally be expected. When the retention proves a persistent one, operation is definitely indicated.

\section{Prostatic Abscess.}

Most cases of prostatic abscess, particularly of the large type, are the result of acute gonococcal prostatitis, though, on occasions, individuals are affected who have not had gonorrhoea. The predominating symptoms are frequency and severe vesical tenesmus. There may be great difficulty with urination and even retention. Differentiating in such circumstances between acute prostatitis and true abscess formation may not be easy. An asymmetrical enlargement in an acutely swollen prostate with a recurring elevation of temperature makes abscess strongly suspect. If a period of observation is required before a definite diagnosis can be made and the urinary retention cannot be overcome by sitting in a hot bath, catheterisation, whilst undesirable, will have to be resorted to. When the diagnosis is established, immediate drainage of the abscess through the perineum is indicated.

\section{Urethral Stricture.}

Acute retention from a urethral stricture is due to neglect of treatment. Contraction gradually occurs, and if the stricture surrounds the urethral canal its lumen becomes almost 
occluded. The slightest mucosal congestion may then suffice to cause a complete obstruction to the passage of urine. The retention is therefore preceded by a gradual diminution in the calibre of the urethral stream, which latterly may only come away a drop at a time. As the vast majority of strictures are a sequel to a previous gonococcal urethritis and develop about three years after the infection, retention from this cause would be expected in a much younger age group than in those due to prostatic obstruction. As, however, an interval of as long as ten years may elapse before the stage of occlusion of the lumen is reached, many of these patients are of the prostatic age. In the circumstances under which the patient is first seen, it may be difficult to obtain an accurate history. The absence of any appreciable enlargement of the prostate on rectal examination will rouse suspicions, though, on the other hand, a stricture of the urethra and a prostatic enlargement may be coincidentally present. If a stricture is not suspected, and an attempt is made to relieve the retention by catheter, the obstruction will be encountered generally at the bulbous urethra, sometimes in the penile portion.

The relief of retention in such cases may prove difficult, but fortified with patience and the proper instruments success can usually be achieved. Immersion in a hot bath is well worth trying as a preliminary measure. If the patient fails to relieve himself in this way, a filiform gum elastic bougie should be passed through the stricture. It may have to be gently manipulated to and from for many minutes before the lumen is engaged, the stricture passed, and the bladder entered. If a number of filiforms are used in the urethra simultaneously, it is usually possible to get one passed. Urine will then, in the course of a few minutes, trickle away alongside the filiform and the bladder will gradually empty itself. If success is only achieved after much difficulty, it is wise to tie in the filiform for twenty-four hours. The patient is thus enabled to pass a small continuous stream, and further dilatation of the stricture can be proceeded with, generally with ease, thereafter.

Although impassable strictures are rare, they are occasionally encountered, and necessitate emptying the bladder by trocar and cannula. With the bladder in a state of distension this can generally be accomplished with local anaesthesia. A small sized trocar is inserted in the mid-line, about two inches above the symphysis. A catheter is passed through the cannula, the latter withdrawn, and the catheter allowed to remain in place for a few days, With the relief of congestion that follows, it will then be possible to pass a filiform and start a gradual dilation of the stricture.

\section{Clot Retention.}

The two most frequent sources of blood clot in the bladder sufficiently marked to cause retention, excluding that which may be a sequel to operations on the prostate, are, bleeding arising from a bladder tumour and that from an enlarged prostate, generally of the very large and soft variety. Clot retention may, however, also follow severe bleeding from the upper urinary tract, due, for example, to a renal tumour. The method employed for relief of the clot is the same whatever the source. It should be borne in mind that although the bladder may be grossly distended, the amount of clot responsible for the hold-up may be comparatively small. In that event the passing of a wide-bore gum elastic or metal catheter and suction through it with a bladder syringe will effectively empty the clot. Alternatively the suction can be carried out through a urethral cannula, and a Bigelow evacuator substituted for the syringe. Large quantities of clot can be expeditiously removed by this method. When the clot has all been evacuated, the bladder is lavaged until a clear return is obtained. An indwelling catheter is then left in the urethra through which the bladder can be washed out, as necessary. If the evacuation has been carried out under anaesthesia, and the patient's condition permits, the opportunity may be taken of making a cystoscopic examination and thereby ascertaining the source and cause of the bleeding.

If the bladder is completely filled with clot, it will not be possible to evacuate it by the above methods, and attempts to do so may be dangerous. Rupture of the already overdistended viscus may result from the instillation of further fluid, incidental to the use of the Bigelow evacuator. In these circumstances evacuation of the clot through a suprapubic cystostomy will probably be necessary, though before resorting to this measure the method described by Riches (I936) for relief of clot retention after transurethral prostatic resection is well worthy of a trial. This consists in injecting into the bladder one ounce of glycerin of pepsin. The catheter through which the injection is made is closed by a spigot and left for half an hour. 
When the spigot is removed the blood-stained urine usually flows out and remaining clots can be withdrawn by suction.

\section{Impacted Urethral Calculus.}

The small stone which has descended from the kidney may be held up in the urethra, after its expulsion from the bladder, and cause a sudden retention. A recent history of renal colic, followed by a quiescent interval or possibly by a period of vesical irritation, is usual. Urethral calculus is the commonest cause of retention in children.

The stone may be impacted in the anterior urethra, where it can generally be palpated, visualised through an anterior urethroscope, or felt by a metal sound or catheter. It is generally possible to remove such stones with the aid of urethral calculus forceps. If, as is frequently the case, the stone is lying just within a narrow meatus, the extraction may be facilitated by a meatotomy, performed under local anaesthesia. Further along the penile urethra, the stone is grasped by well lubricated urethral forceps, the urethra in front of the stone being first distended with novocaine 5 per cent. If a stricture is present in front of the stone an external urethrotomy may be called for.

When the stone is in the posterior urethra, the best plan is to push it back into the bladder. This will immediately relieve the retention, and the stone can be subsequently removed by a cystoscopic lithotrite or rongeur forceps.

\section{Rupture of the Bladder and Urethra.}

Intraperitoneal rupture of the bladder, which is more frequent than the extraperitoneal variety, is characterised by an inability to void, though some blood-stained urine may be passed. immediately after the accident. With an extraperitoneal rupture, which is frequently associated with a fractured pelvis, there may be complete retention, or the patient may be able to pass. some bloodstained urine. Thus, when a bladder injury is suspected, the ability of the patient to pass urine, which is not visibly blood stained, is strong presumptive evidence against there being a rupture. In most instances, injuries involving other structures are present and the 8 vesical symptoms may be overshadowed. In addition, shock is a prominent feature. Catheterisation and the instillation of a measured amount of sterile fluid into the bladder are diagnostic procedures commonly employed. Catheterisation will reveal an empty bladder or the presence? of a small quantity of bloody urine. Failure to recover less than the full amount of a measured quantity of fluid injected through the catheter is suspicious of rupture, though the information thus obtained may be misleading. Intravenous urography can give a positive diagnosis of vesical rupture and reveal the path of the escaping fluid. This procedure may not be practicable on account of the other injuries present, whilst shock may have so impaired the renal function that the contrast solution is excreted in an amount insufficient to outline the bladder shadow and the path of the escaping fluid. As early operation is indicated in the presence of rupture, an exploratory operation should be proceeded with when the diagnosis is doubtful. Operation involves closure of the perforation, drainage of the bladder by suprapubic tube or indwelling catheter, aspiration of the fluid content of the abdomen in intraperitoneal ruptures and drainage of the perivesical space in the extraperitoneal type.

Rupture of the urethra may occur either anterior or posterior to the triangular ligament. The chief symptoms with both varieties are perineal pain with subsequent swelling, bleeding from the meatus, and retention. In all suspected cases an attempt should be made to gently pass a catheter under strict aseptic precautions. If an obstruction is encountered but successfully passed, the catheter should be tied in and retained for several days. If the catheter is completely held up, it can be assumed that a rupture is present and surgical intervention should be immediately planned. Suprapubic cystostomy combined with an anastomosis of the ruptured urethra through the perineum or retrograde catheterisation are the procedures generally indicated. It should be borne in mind that a rupture of the bladder may have coincidentally occurred, and that abdominal or pelvic injuries are commonly present which, as in ruptured bladder, may at first overshadow the urethral injury. If the patient should survive the failure to recognise and treat the latter injury, the possibility of lifelong illhealth from that most intractable of conditions, a traumatic urethral stricture, lies before him. 


\section{Foreign Body.}

Although I have encountered many diverse objects in the bladder, such as a small pin, two hairpins, a kirby grip, slippery elm, a clinical thermometer (in a girl aged twelve), a lead pencil, the end of a rubber catheter, a filiform bougie, and several inches of thin rubber tubing, all but the two latter occurring in female patients, in no instance was there acute retention. An attack might, however, be precipitated by the foreign body impacting at the vesical outlet, especially if a phosphatic incrustation had had time to form around it. In that event there should be no difficulty in passing a catheter, which would either enter the bladder alongside the object, or displace the latter away from its obstructing position. The foreign body should be removed as soon as possible, either by way of the urethra with cystoscopic rongeur forceps, or suprapubically. The route chosen will depend on the size and shape of the object to be removed.

\section{Neurogenic Bladder.}

\section{NON-OBSTRUCTIVE RETENTION}

The vesical dysfunction due to lesions in the nervous system which causes changes in the neuromuscular balance of the bladder is termed "neurogenic bladder." Although such dysfunction may arise from a cerebral or peripheral nerve lesion, the vast majority are due to lesions in the spinal cord, and the term "cord bladder" is commonly used to denote the condition. In civil life injuries to the spine and tabes dorsalis with the various other forms of syphilis of the central nervous system account for the majority of neurogenic bladders, whilst in military practice injuries to the spinal cord are the chief causative factor, a small minority being due to injuries of the brain and the peripheral nerves of the bladder.

When the onset of the central nervous lesion is sudden, as from trauma due to displaced fragments of bone, a penetrating missile, or haemorrhage, the first effect on micturition is retention resulting from spinal shock. This phase may last for two to three weeks, may disappear within twenty-four hours, or may persist for eighteen months or longer. An overflow incontinence follows, until automatic micturition is eventually established. If a urinary infection is present or results, as is so frequently the case from injudicious treatment, the upper urinary tract is early involved, and the kidneys become the seat of a septic pyelonephritis. It is from this complication that most patients die. The objectives to be aimed at therefore in the treatment of retention in a "cord bladder" are, prevention of infection with its inevitable spread to the upper urinary tract, and the encouragement of early automatic micturition.

Opinion is by no means unanimous as to the best means of achieving these objectives, though there is general agreement that intermittent catheterization of such patients is deadly. Manual expression of the urine without catheterisation, a retained urethral catheter, combined with continuous tidal irrigation, and suprapubic drainage through a watertight cystostomy opening, are the three alternative methods which have a place in the care of the paralytic bladder. The reader is referred to the first article in this series for further details.

\section{Post-Operative Retention.}

Post-operative retention may occur after almost any operation, the incidence being rather higher when spinal anaesthesia has been used. Although in. a small number, an obstructive lesion, such as an enlarged prostate is present, but has caused little or no trouble up to the time of operation, the majority of retentions, after operation, are due to neuromuscular disturbances of parasympathetic origin. In dealing with this troublesome condition, every effort should be made to get the patient to resume normal micturition without resorting to the catheter. The patient should first be reassured and encouraged to hope that the urine will soon'be passed naturally. A single dose of hexamine gr. $\mathbf{x v}$ to $\mathbf{x x}$ is prescribed, and if the operative site is no contra-indication, a hot pack may be placed over the hypogastrium and the legs allowed to hang over the side of the bed. If the retention persists, a subcutaneous or intramuscular injection of I c.c. of carbachol can be given in the hope that its stimulating effect on the parasympathetic nerves will result in the bladder evacuating its content. As rather alarming symptoms of vascular disturbances may follow this or analagous parasympathetic stimulants, a $\frac{1}{2}$ c.c. only should be administered to the elderly patient. If these measures fail, the catheter must be employed and urinary antiseptic treatment simultaneously given. 


\section{Hysteria.}

As the nervous system, both voluntary and involuntary, is responsible for maintaining normal vesical function, it is not surprising that persons with neurotic tendencies are liable to functional disturbances of the bladder, and that retention of urine of an hysterical nature may occur. The writer knows of a female patient who was catheterised for several months for complete retention after an appendicectomy operation. Thereafter she became liable to recurring attacks and, although repeated examinations were in turn carried out by some twenty specialists, no one ever discovered any local cause for the malfunction.

It is the absence of any local causative lesion that should arouse suspicion of the true nature of the trouble. In fairness to this unfortunate type of patient, it should be remembered that with most of them, although the urinary difficulty arises from psychological causes, it is by no means imaginary and wilful. It is a wise rule to submit such patients to a careful urological investigation. When the examination has confirmed the absence of any physical abnormality and, at the same time, helped to convince the patient of this, psychotherapy will the easier overcome the mental complexes responsible.

\section{CHRONIC RETENTION}

Chronic urinary retention from whatever cause is an insiduous complaint, and because of this irreparable damage may have occurred before its existence is recognised. Although the following remarks refer in particular to chronic retention due to an obstructing prostate, the principles outlined are applicable to chronic retention arising from any other obstructive lesion at the vesical outlet or urethra.

Once the bladder muscle is unable to extrude all the vesical content, "residual urine" develops. The future of the patient will then depend on whether this remains small in amount or rapidly increases, and on whether or not infection develops. The capacity of the bladder diminishes in proportion to the increase in the amount of the residual, but the patient may not worry about the consequent increase in the urinary frequency. It is quite common for a stage of gross vesical distension to be reached without advice being sought. Even then, the chief symptoms complained of may be lack of vigour, constipation, loss of weight, and dry mouth, the result of back pressure on the upper urinary tract and consequent renal failure. If infection supervenes, fever is added to the syndrome. Thus the clinical picture may be so misleading, that treatment for gastro-intestinal disturbances is not infrequently given over quite a long period until adequate examination reveals the distended bladder. The decrease in the urinary function is frequently accompanied by hypertension and its associated symptoms, particularly in prostatic disease. Although one would normally expect a high incidence of hypertension in patients of this age group, there is evidence that the hypertension is aggravated to an extent more or less proportional to the degree and duration with which the bladder has failed to empty itself. A sudden emptying of the bladder, in such patients, might produce an equally sudden fall in the blood pressure with injurious consequences.

That the chronically distended bladder must not be suddenly emptied, has become a dogma. Numerous theories have been advanced to explain why sudden emptying is dangerous. Those chiefly entertained at present are that a sudden fall in the intrapelvic pressure of the kidney follows, which produces oedema and haemorrhage into the parenchyma, and thus haematuria and renal failure; and that a sudden drop in the intrarenal tension is followed by a fall in the blood pressure, which in turn causes oliguria and anuria. Creevy (I938) compared two series of I20 cases suffering from vesical neck obstruction, all with 500 c.c. or more of residual urine. One group had been treated without regard to the rate of emptying, while the second had been subjected to gradual decompression. Of the first group, $4 \cdot$ I per cent died without other treatment, in the second, the mortality was $4 \cdot 16$ per cent. He therefore concluded that gradual decompression should be abandoned. Whilst agreeing that it is not necessary to carry out a gradual decompression on all patients with chronic retention, the writer considers that this method is valuable and even life-saving in certain instances. The cause of death in many of these patients seems undoubtedly to be due to a septic pyelonephritis, which condition may be precipitated by a too rapid emptying of a chronically distended bladder, particularly when a urinary infection is present. A safe rule, therefore, is to decompress gradually, any chronic retention with a residual of over fifteen ounces when infection has supervened, or when a systolic pressure of $160 \mathrm{~mm}$. or more is present. 
Various methods of decompression can be used, either by catheter via the urethra, or suprapubically. If it seems likely, as judged by the patient's clinical state and kidney functional tests, that the renal efficiency can be sufficiently improved to permit safe operative treatment of the obstructive lesion within a period of about fourteen days, the decompression should be made via the urethra, providing the obstructing lesion permits this. After the catheter is tied in, it can be fitted with a sterile spigot, which is removed every two hours, when eight to ten ounces of urine are allowed to escape. When the bladder is nearly empty, the catheter is connected by tubing to a bottle containing an antiseptic solution. A method of obtaining a controlled rate of decompression, which I particularly favour, is that of connecting the catheter by tubing to a receptacle at the side of the bed, interposing a glass interceptor, such as is routinely used for intravenous drip infusions and allowing the urine to be evacuated at the rate of 30 to 40 drops per minute. When the patient's condition is such that at least several weeks of bladder drainage will be required to adequately improve on the renal function and general state, the decompression should be carried out through the suprapubic route from the outset. This applies in particular to the prostatic with a long-standing gross distension, or retention with overflow, an infected urine, and a blood urea of perhaps over roo mg. per cent. A watertight self-retaining suprapubic tube can be inserted into such a distended bladder under local anaesthesia, without losing more than a few drops of urine in the process, and the bladder slowly emptied by the same methods employed with a tied-in urethral catheter. Simultaneous with the decompression, diuresis is enforced, a minimum fluid intake of 5 pints daily being the objective. If nausea, or inability of the patient to co-operate because of uraemic manifestations, precludes the oral route, a continuous intravenous infusion should be started immediately and, providing the diuretic response is satisfactory, maintained until the patient can drink copiously. Sodium sulphate solution, saline, and saline with glucose are the most useful preparations for promoting renal secretion.

Decompression and drainage of the bladder in chronic vesical distension is a preliminary measure to the conditioning of the patient for the treatment of the underlying lesion and not an end in itself. It is true that some may have to be content with permanent suprapubic drainage, as for example the prostatic with an advanced and irreparable renal impairment, or with serious cardio-vascular degeneration. If, after about two months' drainage, such patients are still deemed unfit for operation, further improvement can seldom be expected, and any major operative intervention will be a hazard. But the majority have a happier outlook. With the improvement that generally follows free drainage, the obstructive lesion can be safely dealt with and normal micturition with complete emptying of the bladder restored.

REFERENCES

C. D. CREEVY. J. Urolg., 1938., 39, 4 . E. W. RICHES. B.M.J., I936, 1, 578 .

\title{
INCONTINENCE OF URINE IN WOMEN*
}

\author{
By E. CATHERINE LEWIS, M.S., F.R.C.S.
}

(Surgeon to the Royal Free Hospital; Senior Surgeon and Urologist to the South London Hospital)

Few disabilities are so uncomfortable to a woman as urinary incontinence. She is constantly aware of the characteristic odour, is unable to keep her clothes fresh and is much troubled by vulval soreness or pruritus consequent upon the trickling urine. The sufferer becomes selfconscious and disinclined to mix with others or to go to places of amusement, and life becomes a burden to her. Successful treatment is an inestimable boon.

\section{TYPES OF INCONTINENCE}

I. False incontinence. In this condition, which might equally well be termed imperious micturition, the urine is forced out through the normal channel by a sudden contraction on the part of the bladder musculature sufficiently forcible to overcome the resistance of a normal sphincter; it is one of the variable symptoms of the so-called "irritable bladder." The patient

* Being a reprint from an earlier Journal.-Ed. 